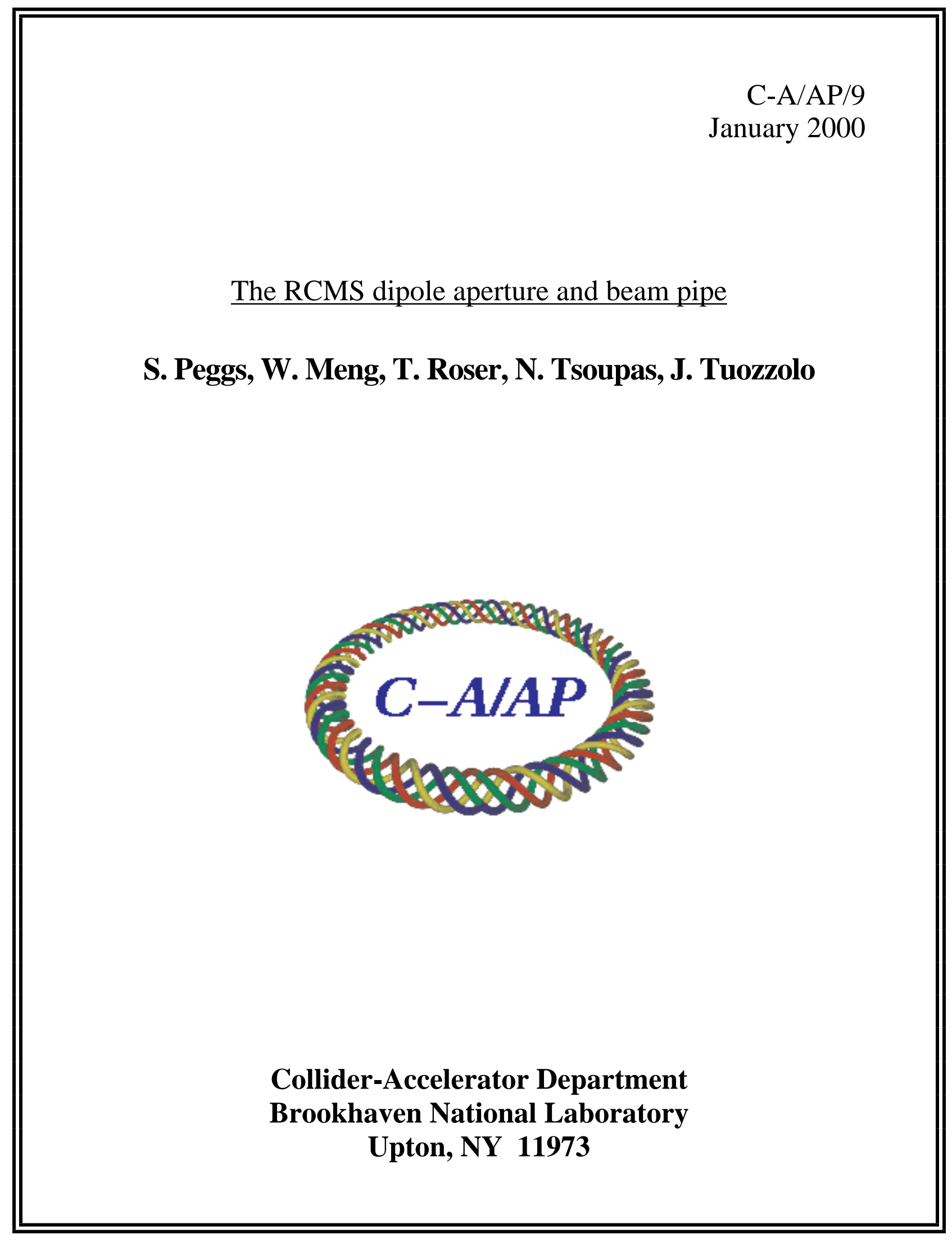




\title{
The RCMS dipole aperture and beam pipe
}

\author{
S. Peggs, W. Meng, T. Roser, N. Tsoupas, J. Tuozzolo
}

\section{Contents}

1 Abstract 1

2 Reducing the dipole aperture $\quad 3$

2.1 Beam sagitta in the dipole and in the beam pipe . . . . . . 3

3 Beam pipe eddy currents 5

3.1 Dipole field distortions ................ 5

3.2 Beam pipe heating ................. 5

4 Beam pipe impedance $\quad 6$

4.1 The longitudinal microwave instability . . . . . . . . . . . 7

\section{Abstract}

The RCMS dipole aperture is reduced to $60 \times 30 \mathrm{~mm}(\mathrm{H} \times \mathrm{V})$ from the value of $80 \times 40 \mathrm{~mm}$ used in the Pre-Conceptual Design Report (PCDR) [1].

The cycling frequency is increased to $60 \mathrm{~Hz}$ from the PCDR value of $15 \mathrm{~Hz}$.

The chevron dipole is constructed from two straight stacks of laminations, reducing the beam sagitta and providing the same edge focusing in both planes.

The dipole beam pipe is made from four straight sections of circular Inconel beam pipe with a radius of about $15 \mathrm{~mm}$.

The optimum beam pipe thickness is between $0.5 \mathrm{~mm}$ and $1.0 \mathrm{~mm}$, balancing the twin desires to keep beam pipe heating less than $300 \mathrm{~W} / \mathrm{m}$, and to keep the beam pipe opaque to wake fields at frequencies of around $1 \mathrm{MHz}$ and higher.

The dipole field perturbation from eddy currents induced in the beam pipe is negligible, and the sextupole perturbation is zero (for a circular pipe).

The RCMS is very stable against collective instabilities. 


\begin{tabular}{|c|c|c|c|}
\hline Parameter & Loma Linda & $\begin{array}{l}\text { RCMS } \\
\text { (PCDR) }\end{array}$ & Comment \\
\hline Protons per pulse & $<3 \times 10^{10}$ & $3 \times 10^{9}$ & 1993 'typical' \\
\hline Pulse rate $[\mathrm{Hz}]$ & .45 & 15 & \\
\hline Patient rate $[1 /$ day $]$ & 125 & & 16 hour days \\
\hline Total power $[\mathrm{kW}]$ & 370 & $>182$ & \\
\hline Circumference [m] & 20.053 & 28.6 & \\
\hline Energy range $[\mathrm{MeV}]$ & $2-250$ & $7-270$ & Inject to top \\
\hline Injection beta gamma & .065 & .122 & \\
\hline B field $[\mathrm{T}]$ & $0.1-1.5$ & $.23-1.5$ & Min - Max \\
\hline Full dipole gap [mm] & $200 \times 50$ & $80 \times 40$ & $\mathrm{H} \times \mathrm{V}$ \\
\hline Full vac chamber [mm] & $96 \times 50$ & & $\mathrm{H} \times \mathrm{V}$ \\
\hline Good field ap. [mm] & $50 \times 50$ & & measured: sext \\
\hline$\Delta p / p$ RF bucket & \pm .0044 & \pm .004 & \\
\hline$\Delta p / p$ beam FWHM & .007 & .0046 (total) & at injection \\
\hline Dispersion max $[\mathrm{m}]$ & 9.6 & 2.18 & \\
\hline $\mathrm{H}$ beam size FWHM $[\mathrm{mm}]$ & 60.0 & 10.0 & LL measured \\
\hline RMS emittance $[\mu \mathrm{m}]$ & $0.11 \times 0.10$ & $0.3 \times 0.3$ & $\mathrm{HxV}$ normalized \\
\hline Beta max $[\mathrm{m}]$ & $6.0 \times 3.2$ & $3.4 \times 4.2$ & $\mathrm{HxV}$ \\
\hline Beta beam size rms $[\mathrm{mm}]$ & $3.2 \times 2.2$ & $2.9 \times 3.2$ & $\mathrm{HxV}$ injection \\
\hline Tune $(H, V)$ & $.600,1.317$ & $3.25,4.85$ & \\
\hline Transition gamma & .583 & 2.39 & \\
\hline Nat. chrom. $(\mathrm{H}, \mathrm{V})$ & $-.61,-1.25$ & $-2.24,-2.71$ & $\mathrm{H}, \mathrm{V}$ design \\
\hline Nat. chrom. & -22 & & field calc \& msmt \\
\hline RF harmonic & 1 & 1 & \\
\hline $\mathrm{RF}$ voltage $[\mathrm{kV}]$ & $<.3$ & 4.5 & \\
\hline RF frequency $[\mathrm{MHz}]$ & $.974-9.174$ & $1.27-6.61$ & \\
\hline
\end{tabular}

Table 1: A comparison of parameters between Loma Linda as built and RCMS numbers as originally reported in the Pre Conceptual Design Report. 


\section{Reducing the dipole aperture}

Table 1 shows a side-by-side comparison of Loma Linda Synchrotron (LLS) parameters, many of them measured [2, 3, 4, 5], and the Rapid Cycling Medical Synchrotron (RCMS) design, as originally described in the PCDR [1].

A comparison of the parameters shows that it is possible to reduce the dipole aperture below the PCDR value of $80 \times 40 \mathrm{~mm}(\mathrm{H} \times \mathrm{V})$ :

- Maximum dispersion is $9.6 \mathrm{~m}$ in the LLS, but only $2.2 \mathrm{~m}$ in the RCMS.

- Momentum width is approximately the same value, $\Delta p / p \simeq \pm .004$, in both machines.

- Horizontal beam size at injection is dominated by momentum spread in both machines.

- Horizontal beam size (FWHM) is measured as $60 \mathrm{~mm}$ in LLS, but is only $10 \mathrm{~mm}$ in RCMS.

- Normalized emittance measured in LLS is about $\epsilon \simeq 0.1 \mu \mathrm{m}$, suggesting that the RCMS value of $0.3 \mu \mathrm{m}$ is conservatively large.

- Vertical betatron beam size is measured in LLS at about $3 \mathrm{~mm}$, and is less than $4 \mathrm{~mm}$ in RCMS.

Putting all this together, it is reasonably prudent to reduce the dipole aperture to $60 \times 30 \mathrm{~mm}$, provided beam sagitta can be handled.

\subsection{Beam sagitta in the dipole and in the beam pipe}

Figure 1 shows that the beam sagitta in the dipole aperture is reduced by a factor of 4 to $8.2 \mathrm{~mm}$ when the dipole is constructed as a "chevron", in two straight rectangular pieces. This permits a dipole aperture width of $60 \mathrm{~mm}$. It has the additional advantage of providing the same edge focusing in both planes - so that focusing and defocusing quadrupoles have approximately the same strength.

A beam sagitta of $8.2 \mathrm{~mm}$ in the beam pipe is still uncomfortably large, if the beam pipe is circular with a diameter of $30 \mathrm{~mm}$, and the horizontal beam size (FWHM) is approximately $10 \mathrm{~mm}$. The beam pipe sagitta is therefore reduced by another factor of 4 , to $2.0 \mathrm{~mm}$, by constructing the dipole beam pipe from four straight sections, instead of two. A circular beam pipe of $30 \mathrm{~mm}$ diameter then has an acceptable horizontal physical aperture of $28 \mathrm{~mm}$ (minus the thickness of the beam pipe wall). 


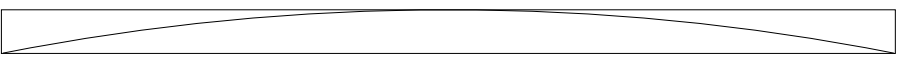

sagitta $=32.5 \mathrm{~mm}$

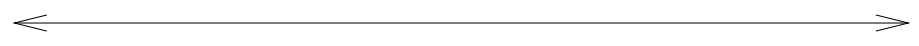

magnetic length $=661 \mathrm{~mm}$

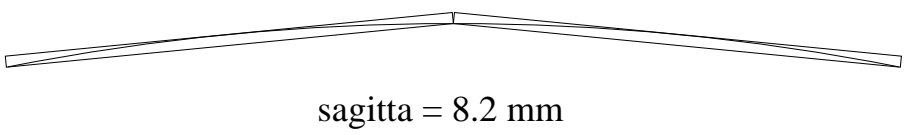

Four piece beampipe sagitta $=2.0 \mathrm{~mm}$

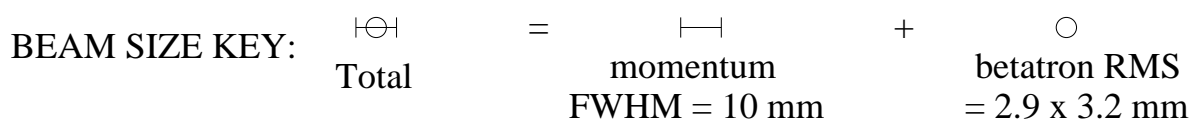
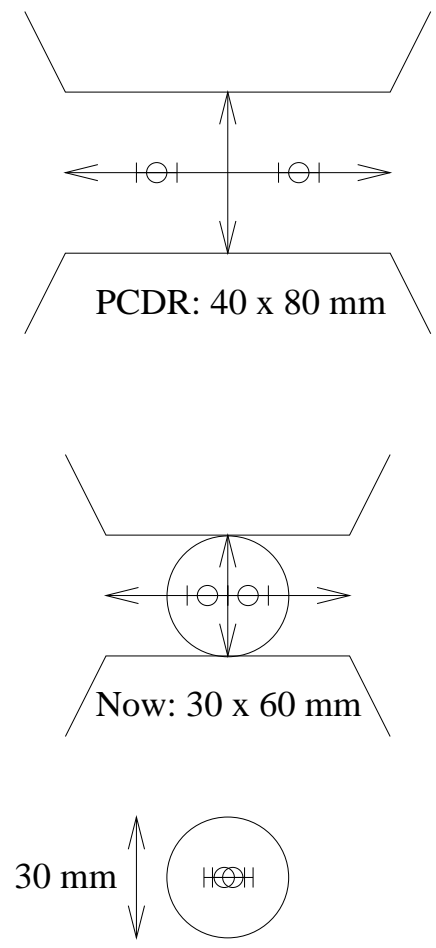

$=2.9 \times 3.2 \mathrm{~mm}$

Figure 1: Beam sagitta in the RCMS dipole and in the beam pipe. The laminations of the dipole described in the PCDR are stacked in a rectangular jig, with a horizontal beam sagitta of $32.5 \mathrm{~mm}$ inside a straight aperture of $40 \mathrm{x}$ $80 \mathrm{~mm}(\mathrm{~V} \times \mathrm{H})$. The beam sagitta in the aperture of a chevron dipole - with two rectangular segments - is reduced to $8.2 \mathrm{~mm}$, enabling the aperture to be reduced to $30 \times 60 \mathrm{~mm}(\mathrm{~V} \times \mathrm{H})$. The beam sagitta relative to the center of the beam pipe is reduced even further - to only $2.0 \mathrm{~mm}$ - if the beam pipe is constructed from four straight segments, instead of two. There is then ample physical aperture for the beam inside a $30 \mathrm{~mm}$ diameter pipe. 


\section{Beam pipe eddy currents}

Eddy currents in the dipole beam pipe are driven by the oscillating part of dipole field, given by

$$
B_{\text {drive }}=B_{0} \sin (\omega t)
$$

where $B_{0} \approx 0.75 \mathrm{~T}$ if the peak field is $1.5 \mathrm{~T}$. At a horizontal distance $x$ from the pipe centerline, and when the skin depth is much greater than the pipe thickness, the eddy current density is

$$
j=j(x)=\sigma \dot{B} x
$$

where $\sigma$ is the conductivity of the pipe, and $\dot{B}$ is the rate of change of dipole field. These eddy currents distort the dipole magnetic field (in time and space), and also cause beam pipe heating. With a $30 \mathrm{~mm}$ diameter Inconel beam pipe the cycling frequency $f_{A C}$ is increased to $60 \mathrm{~Hz}$ from the $15 \mathrm{~Hz}$ value quoted in the PCDR.

\subsection{Dipole field distortions}

A thin circular beam pipe has a $\cos \theta$ eddy current distribution which only distorts the dipole field - there is no sextupole component, et cetera. According to Chao and Tigner [6] (p. 264) a beam pipe of radius $b$ and thickness $t$ causes the net dipole field to become

$$
B=\frac{B_{0}}{\sqrt{1+\omega^{2} \tau^{2}}} \sin \left(\omega t-\tan ^{-1}(\omega \tau)\right)
$$

where

$$
\tau=\mu_{0} \sigma b t / 2
$$

Assuming an Inconel 625 or X750 beam pipe, with $b=15 \mathrm{~mm}$ and a resistivity of $\rho=1 / \sigma \approx 1.25 \mu \Omega-\mathrm{m}$, then

$$
\tau=7.5 t[\mathrm{~mm}] 10^{-6}[\mathrm{~s}]
$$

Combining this with an angular frequency of $\omega=2 \pi f_{A C}$ gives

$$
\omega \tau=0.0028 \frac{f_{A C}[\mathrm{~Hz}]}{60} t[\mathrm{~mm}]
$$

The dipole field is barely perturbed by a $1 \mathrm{~mm}$ thick beam pipe.

\subsection{Beam pipe heating}

The instantaneous heating power per unit volume of beam pipe is

$$
\frac{d P}{d V}=\frac{j^{2}}{\sigma}=\sigma \dot{B}^{2} x^{2}
$$


so that the instantaneous power per unit length is

$$
\frac{d P}{d s}=\pi \sigma \dot{B}^{2} b^{3} t
$$

and the average power per unit length is

$$
\left\langle\frac{d P}{d s}\right\rangle=2 \pi^{3} f_{A C}^{2} \sigma B_{0}^{2} b^{3} t
$$

With an Inconel pipe of radius $b=15 \mathrm{~mm}$, and an amplitude of $B_{0}=0.75 \mathrm{~T}$, this power becomes

$$
\left\langle\frac{d P}{d s}\right\rangle=339[\mathrm{~W} / \mathrm{m}] \quad\left(\frac{f_{A C}[\mathrm{~Hz}]}{60}\right)^{2} t[\mathrm{~mm}]
$$

Chao and Tigner [6] (p. 315) state that the temperature rise above an ambient temperature of $300 \mathrm{~K}$ due to the free convection of air over a vertical panel of height $h$ is

$$
\Delta T=0.454\left\langle\frac{d P}{d s}\right\rangle^{4 / 5} h^{-3 / 5}=300\left(\left\langle\frac{d P}{d s}\right\rangle / 339\right)^{4 / 5}[\mathrm{~K}]
$$

where an effective vertical height of $h=\pi b=47 \mathrm{~mm}$ has been assumed. In practice the nearby magnet poles impede the free flow of air over the beam pipe, but also act as substantial heat sinks.

From the perspective of beam pipe heating alone, it is desirable to use an Inconel beam pipe thinner than $t=1 \mathrm{~mm}$. This is not a challenge to mechanical stability, since pipes thinner than $0.5 \mathrm{~mm}$ are strong enough to withstand atmospheric pressure, even without the use of reinforcing ribs.

\section{Beam pipe impedance}

The skin depth for Inconel is

$$
\delta_{s}=\frac{1}{\sqrt{\sigma f \pi \mu}}=\frac{0.56}{\sqrt{f[\mathrm{MHz}]}}[\mathrm{mm}]
$$

where it is assumed that the relative permeability $\mu_{r}=\mu / \mu_{0}=1$. The critical frequency above which wake fields will not penetrate a pipe of thickness $t$ is

$$
f_{c}=\frac{1}{\sigma \pi \mu t^{2}}=\frac{0.314}{(t[\mathrm{~mm}])^{2}}[\mathrm{MHz}]
$$

This frequency is to be compared with that of the RF system, which is an approximate lower bound on the power spectrum of the wake fields of the bunch. 
The radio frequency is the same as the revolution frequency $f_{r e v}$, since the harmonic number $h=1$. It increases from $1.27 \mathrm{MHz}$ at injection to $6.61 \mathrm{MHz}$ at top energy. Thus, an Inconel beam pipe must be thicker than about $t=0.5$ $\mathrm{mm}$ for almost no wake fields to penetrate during injection.

If the same Inconel beam pipe of radius $b=15 \mathrm{~mm}$ is used around the entire circumference of the RCMS, then the longitudinal resistive wall impedance at a frequency $f$ well above $f_{c}$ is given by

$$
\frac{Z_{\|}}{n}(f)=\frac{\mu_{r} Z_{0}}{2 b} \delta_{s}=\frac{7.0}{\sqrt{f[\mathrm{MHz}]}}[\Omega]
$$

where $Z_{0}=377 \Omega$ is the impedance of free space, and $n$ is the mode number (so $f=n f_{\text {rev }}$ ). Similarly the transverse resistive wall impedance is

$$
Z_{\perp}(f)=\frac{\mu_{r} Z_{0} R}{b^{3}} \delta_{s}=\frac{0.51}{\sqrt{f[\mathrm{MHz}]}}[\mathrm{M} \Omega / \mathrm{m}]
$$

where $R=C / 2 \pi=4.55 \mathrm{~m}$ is the average radius of the RCMS.

\subsection{The longitudinal microwave instability}

Following Chao and Tigner [6] (p. 118), the Boussard criterion for stability against the longitudinal microwave instability is written

$$
\frac{Z_{\|}}{n} \leq F^{\prime} \frac{m_{p} c^{2}}{e} \frac{|\eta| \gamma}{I_{b}}\left(\frac{\Delta p_{F W H M}}{p}\right)^{2}
$$

where $F^{\prime} \approx 1$ is a form factor depending on the details of the bunch distribution, and $\eta=1 / \gamma_{T}^{2}-1 / \gamma^{2}$ is the slip factor. The peak gaussian bunch current is

$$
I_{b}=N_{B} e f_{r e v} \frac{C}{\sqrt{2 \pi} \sigma_{s}}
$$

where $C$ is the circumference, and the rms length $\sigma_{s}$ is always at least $3 \mathrm{~m}$ in the RCMS. Table 2 shows that the threshold impedances are orders of magnitude larger than that of the beam pipe when a nominal proton bunch of $N_{B}=$ $3.3 \times 10^{9}$ is injected at a current of $2.1 \mathrm{~mA}$.

The low intensity strong focusing RCMS is very stable against the longitudinal microwave instability in particular, and against collective instabilities in general. 


\begin{tabular}{|lcc|}
\hline Parameter & Injection & Top energy \\
\hline & & \\
Slip factor $\eta$ & -0.81 & -0.43 \\
Lorentz $\gamma$ & 1.008 & 1.288 \\
Bunch peak current $I_{b}[\mathrm{~mA}]$ & 2.1 & $\sim 1$ \\
Relative momentum spread, $\Delta p_{F W H M} / p$ & 0.006 & 0.001 \\
& & \\
Threshold impedance $Z_{\|} / n[\Omega]$ & $1 \times 10^{7}$ & $5 \times 10^{5}$ \\
\hline
\end{tabular}

Table 2: Parameters in the calculation of the maximum impedance for stability against the longitudinal microwave instability.

\section{References}

[1] Pre-Conceptual Design of a Rapid Cycling Medical Synchrotron, S. Peggs editor, BNL C-A/AP/6, October 1999.

[2] Technical Assessment of the Loma Linda University Proton Therapy Accelerator, F. Mills chairman, FNAL TM-1656, October 1989.

[3] A Performance Study of the Loma Linda Proton Medical Accelerator, G. Coutrakon et al, October 93.

[4] Design Considerations for Medical Proton Accelerators, G. Coutrakon, J. Slater, and A. Ghebremedhin, Particle Accelerator Conference, New York, 1999.

[5] Multi-Station Proton Beam Therapy System, F. Cole et al, US Patent 4,870,287, September 1989.

[6] Handbook of Accelerator Physics and Engineering, A. Chao and M. Tigner, World Scientific, 1st edition, 1998. 